\title{
Research on 3D Wireless Sensor Networks Energy Saving Routing Algorithm
}

\author{
Zhanguo $\mathrm{LI}^{1, \mathrm{a}}$, Jianxi WANG ${ }^{1}$, Xingwei WANG ${ }^{2}$ \\ 1. School of Software Engineering, Pingdingshan University, Pingdingshan 467002, China \\ 2. College of Information Science and Engineering, Northeastern University, Shenyang 110819, \\ China \\ aemail: lizhanguo01@sohu.com
}

Keywords: Three-dimensional Wireless Sensor Networks(3d WSN); Energy Angle; Routing Algorithm; Clustering; Energy Saving

\begin{abstract}
In this paper, the energy saving 3D wireless sensor networks Iterative Split Clustering Energy Angle Routing (ISC-EAR) algorithm will be proposed and also discuss the design and calculation process in theory. The simulating calculation under different topology, compared with the baseline algorithm the ISC-EAR routing algorithm, has better energy saving effect, can effectively prolong the survival time of the network.
\end{abstract}

\section{Introduction}

In recent years, 3d wireless sensor network technology has been widely applied in respect of the underground sensor network, underwater sensor network, space sensor network, smart home and the field of structural health monitoring etc. Generally, main research interests include: network coverage, localization algorithm, data aggregation, and routing algorithm, etc. This paper mainly study on 3d wireless sensor network routing algorithm.

There has been a lot of research results in terms of 3d wireless sensor network routing algorithm research. In respect of the routing algorithm in 3d wireless sensor network (3d WSN), what the major researches focus on is the routing algorithm based on clustering. Its objective is to minimize the power consumption, and to improve the use time of the network. Rahman[1] is proposed a mathematical model is proposed to find out the energy consumption rate and to distribute the optimum number of sensor node in each corona according to energy consumption rate. An algorithm is proposed to distribute the optimum number of sensor nodes in corona-based networks. A cooperative group-based network reduces the number of the messages transmitted inside the network was proposed in [2], which reduction of energy consumed by the whole network, and increase of the network lifetime.

This paper proposes an Iterative Split Clustering Energy Angel Routing (ISC-EAR) algorithm, which reduction of energy consumed by the whole network, and increase of the network lifetime. Using the simulation calculation on different network topology, ISC-EAR Routing algorithm with the international benchmark algorithm IGreedy [3] compared various technical indicators have very good performance, has good advancement and research value.

\section{Iterative Split Clustering Energy Angel Routing, ISC-EAR}

\subsection{ISC - EAR Design Idea}

With a goal of saving energy and extending the work time, a 3d wireless sensor network routing algorithm is proposed, the basic idea of the algorithm is, firstly, to select the candidate of nodes which meet the user bandwidth demand from all the neighbor nodes in the radius of the node sensation, secondly, considering the distance of current node to neighbor node space and the angle of choice of the next-hop node, then in according to the node and the distance to the neighbor node sends the ratio of the energy consumption, at the same time, and set different weight of these two factors, to ensure that the selected node sends furthest distance and minimum energy consumption, 
deviating from the destination node angle factors to prevent the node too far.

\subsection{ISC-EAR Operation Rules}

The ISC-EAR algorithm main operation includes the following seven parts:

1) The determination of candidate set of nodes

Node sensing radius or communication radius can directly communicate node called the neighbor node, the node and link bandwidth between neighbor nodes is greater than the bandwidth needed for the user, the neighbor nodes can be used as candidates for the next-hop node, all can communicate directly with the current node and meet the demand of the user bandwidth under the nodes of a candidate set of nodes.

2) Determine the Angle of vector space

Set up the current node coordinates $C\left(x_{c}, y_{c}, z_{c}\right)$, destination node $D\left(x_{d}, y_{d}, z_{d}\right)$, and neighbor node $N\left(x_{n}, y_{n}, z_{n}\right)$, then the vectors of the current node and destination node is expressed as:

$\vec{a}=\left(x_{d}-x_{c}, y_{d}-y_{c}, z_{d}-z_{c}\right)$

Vectors of the current node and neighbor node is as follows:

$\vec{b}=\left(x_{n}-x_{c}, y_{n}-y_{c}, z_{n}-z_{c}\right)$

The cosine value of space angle $\beta$ made by current node, destination node and neighbour node is shown as (use current node as vertex):

$$
\cos \beta=\frac{\left(x_{d}-x_{c}\right)\left(x_{n}-x_{c}\right)+\left(y_{d}-y_{c}\right)\left(y_{n}-y_{c}\right)+\left(z_{d}-z_{c}\right)\left(z_{n}-z_{c}\right)}{\sqrt{\left(x_{d}-x_{c}\right)^{2}+\left(y_{d}-y_{c}\right)^{2}+\left(z_{d}-z_{c}\right)^{2}} \times \sqrt{\left(x_{n}-x_{c}\right)^{2}+\left(y_{n}-y_{c}\right)^{2}+\left(z_{n}-z_{c}\right)^{2}}}
$$

The spatial angle $\beta$ come from the inverse cosine function is expressed as

$$
\beta=\arccos (\cos \beta)
$$

3) Calculate consumption of nodes sending energy

According to the model, the $E_{T X}$ (consumption of energy sending of node) is shown as

$$
E_{T x}(k, d)=E_{T x-\text { elec }}(k, d)+E_{T x-a m p}(k, d)=E_{\text {elec }} * k+\varepsilon_{\text {amp }} * k * d^{\alpha}=\left\{\begin{array}{l}
E_{\text {elec }} * k+\varepsilon_{\text {fs }} * k * d^{2}, d<d_{0} \\
E_{\text {elec }} * k+\varepsilon_{\text {mp }} * k * d^{4}, d \geq d_{0}
\end{array}\right.
$$

$E_{\text {elec }}$ is the energy consumption of transmitting and receiving circuits, $k$ as the packet size, $\varepsilon_{a m p}$ for energy consumption of power amplifier, when the transmission distance is less than $d_{0}$, then $\alpha=2$, through using the free space model, namely the $\varepsilon_{a m p}=\varepsilon_{f s}$. When the transmission distance is greater than $d_{0}$, then $\alpha=4$, and using the multipath fading model, namely the $\varepsilon_{a m p}=\varepsilon_{m p}$ 。

4) Determine the next-hop node

Total energy consumption of Node includes sending and receiving energy consumption, the sending energy consumption in total energy consumption accounts for a large proportion, and energy consumption of nodes sending related to the distance, so in order to decrease the total energy consumption of node, guarantee the sending distance as far as possible to cost the least energy consumption when choosing the next hop node. In addition, in order to prevent the next hop node deviating from the destination node, it is a need to choose the smallest space vector angle made by current node, destination node and neighbor node with using current node as vertex, basically, choose the candidate nodes which is closest to the destination node as the next hop node. Then taking the above two factors into consideration, setting the different weights, to select the best the next hop node from the collections of candidate nodes.

Select the next hop node(Next hop, NT) on a basis of expression:

$$
N T=w_{1} * \frac{d}{E_{T X}}+w_{2} * \frac{1}{\beta}
$$

$w 1$ and $w 2$ are the sum of the two weights here, and $w_{1}+w_{2}=1, d$ means the distance from the 
current node to the neighbor node, $E_{T X}$ is the energy needed for launching to the next hop node, $\beta$ is the spatial angel made by current node, neighbor node and destination node (current node is the vertex).

To sum up, the greater the $d / E$ is, the less energy cost by launching the most distant way; the greater $1 / \beta$ is, the smaller space Angle is, then it is closer to the destination node, so the most distant way in the $d / E$ is larger, shows the minimum energy consumption; the $1 / \beta$ greater, the space angle is smaller, the more close to the destination node.

5) Forming path

In order to prevent the loop, set the selected node Selected nodes set, each node in the process of find a way can only be selected once. Once the node is selected, each selected the next-hop node stored in the Rout path set in turn. After choosing the next-hop the node loses the qualification of the selected. In addition, if the current node can't find meet the requirements of the next-hop node, from the current node, pops up in Rout path set to choose NT value times bigger as the next-hop node, and mark the current node state $=0$, next time don't choose this node as the next-hop node.

6) Identify nodes remaining energy

According to the formula (6) the residual energy of each node is calculated, update the residual energy of nodes and run the ISCA algorithm to select cluster head.

$$
E_{R}=E-E_{T x}-E_{R x}
$$

The $E_{R}$ refers to the residual energy of nodes, the current energy $E$ for the node, $E_{T x}$ for energy consumption of nodes to send data calculated by formula $4, E_{R x}$ for receiving data node energy consumption calculated by the formula 7 .

$$
E_{R x}(k)=E_{T x-\text { elec }}(k)=k^{*} E_{\text {elec }}
$$

\section{7) Data aggregation}

Numerous nodes in wireless sensor network data, there may be redundant data collected from different nodes information, and these redundant data information in the process of transmission will be unnecessary energy waste, so need to cluster of cluster nodes in data aggregation processing, to achieve the purpose of reducing energy consumption data transmission. Each cluster head of data fusion rate was $70 \%[4]$, calculation formula:

$$
D_{\text {after }}=D \times 70 \%
$$

\section{The Simulation Analysis}

To measure the design in this paper the performance of 3d wireless sensor network routing algorithm, this article using the $\mathrm{C} / \mathrm{C}++$ language to realize the simulation calculation algorithm, and compared with the international benchmark algorithm IGreedy contrast.

\subsection{Benchmark Algorithm}

Greedy algorithm is a classic of 3d wireless sensor network routing algorithm, the algorithm based on greedy algorithm proposed IGreedy-PAGR (Power adjusted greedy algorithm with optimal transmission range and threshold) [3] based on the optimal transmission radius and adjustable threshold energy greedy routing algorithm.

Igreedy algorithm using adaptive power control transmission radius instead of the traditional constant transmission radius, by adjusting the transmission power nodes to reduce the energy consumption of transmitting data to send to achieve the goal of energy saving. Igreedy algorithm of initial setting node Transmission radius for the Optimal Transmission radius OR (Optimal Transmission Range), using the greedy routing algorithm for data forwarding path, known as the OR model; When meet routing hole (Local Minimum), in order to find the next hop node, the Transmission radius increases to MR (Minimum Transmission Range), continue to use the greedy routing algorithm for path, called MR mode. In order to accurately determine when to use the OR or MR mode, algorithm to set a threshold parameter $\mathrm{DR}=4$ node degree. The initial use OR mode select neighbor nodes, when the node degrees down to less than the threshold value of DR, use MR 
mode. When the node degrees rebounded more than the threshold, in return for the OR model, so repeatedly, until you find destination node failure OR cannot find a neighbor node algorithm.

\subsection{Topology Case}

In order to test the design of the performance of the $3 \mathrm{~d}$ wireless sensor network routing algorithm in this paper, respectively using the random topology, average topology and mine topology routing algorithm simulation, and compared with algorithm based on the benchmark analysis.

Random topology side within 100 m cube randomly deployed 100 nodes, three-dimensional coordinates of the nodes randomly generated by computer.

100 nodes in average topology approximation evenly distributed in the side length of $100 \mathrm{~m}$ cube area. First will be evenly divided into 64 small cube, the cube and the nodes are placed in the center of the each small cubes. The remaining 36 nodes deployed in the six surfaces of the cube, each place six surface, by mathematical formula can be calculated from the $3 \mathrm{~d}$ coordinates of 100 nodes.

Mine topology using the underground tunnel topology, the length and width is $5 \mathrm{~m}$, the height is $100 \mathrm{~m}$, sink node is located in the surface coordinates of $(0 \mathrm{~m}, 0 \mathrm{~m}, 0 \mathrm{~m})$. In order to reduce the average node Energy consumption and prolong network life time, this paper uses Energy efficient sensor node deployment algorithm EESP (Energy efficient sensor placement)[5], the algorithm based on node energy consumption model, deduced the formula of the distance between the nodes $d i$, as shown in formula (9).

$$
d_{i}=\frac{L}{\sqrt[3]{i} \times \sum_{i=1}^{n} \sqrt[3]{\frac{1}{i}}}
$$

$L$ is the height of the network topology area, $n$ is the number of sensor nodes in network topology, $d_{i}$ for the node $v_{i+1}$ and the distance between the $v_{i}$. The sensor nodes are more close to the sink node, deployment more densely populated.

\section{Test results}

In order to evaluate the performance of the algorithm proposed in this paper, at the random topology, average topology and mine (tunnel) topology for testing. From effective alive nodes, the network survival time, the network energy consumption and average energy consumption of node based on the four aspects of the performance evaluation of energy consumption.

\subsection{Alive nodes}

Number of alive nodes in network is able to have enough energy, to send data from a source to sink node. The alive nodes reflect the network survival time, the more alive nodes information integrity is higher, alive node is less, cannot acquire network information.

In the random topology, average topology and mine (tunnel) topology under three kinds of network topology, ISC-EAR routing algorithm and the Igreedy routing algorithm test results for number of alive nodes as shown in table 1

Table 1 Number of alive nodes

\begin{tabular}{|c|l|c|c|c|c|}
\hline Routing algorithm & Simulation time(sec) & $\mathbf{1 0 0 0}$ & $\mathbf{2 0 0 0}$ & $\mathbf{3 0 0 0}$ & $\mathbf{4 0 0 0}$ \\
\hline \multirow{3}{*}{ ISC-EAR } & Mine topology & 98 & 81 & 65 & 41 \\
\cline { 2 - 6 } & Average topology & 98 & 76 & 60 & 37 \\
\cline { 2 - 6 } & Random topology & 98 & 73 & 46 & 27 \\
\hline \multirow{3}{*}{ IGreedy } & Mine topology & 95 & 75 & 55 & 27 \\
\cline { 2 - 6 } & Average topology & 97 & 69 & 49 & 13 \\
\cline { 2 - 6 } & Random topology & 96 & 66 & 27 & 0 \\
\hline
\end{tabular}

From table 1, with the increase of the simulation time, node number is decline trends at three network topology. Different topology at the same time simulation contrast, mine topology nodes is 
the most effective survival. The same topology at the same time simulation comparison, the ISC-EAR algorithm is more effective than benchmark algorithm for node number. For example, in the simulation time is 4000 seconds, benchmark algorithm of nodes have all died at the random topology, the ISC-EAR algorithm have 41 node alive at the mine topology, greatly prolongs the network survival time.

\subsection{Network lifetime}

The lifetime of wireless sensor network is run from the network start transmitting data to the normal operation of the network, to provide users with the duration of the monitoring environmental information so far. In this paper, using to measure the first nodes death time, the half nodes death time and the all nodes death time. The first nodes death time is used to measure the balance of routing algorithm. Half nodes death time is used to measure the effectiveness of the proposed routing algorithm. All nodes death time is used to measure the longest survival time of the network.

Table 2 shows at three kinds of network topology the ISC-EAR routing algorithm and the Igreedy routing algorithm test results for network lifetime.

Table 2 Network lifetime

\begin{tabular}{|c|l|c|c|c|}
\hline $\begin{array}{c}\text { Routing } \\
\text { algorithm }\end{array}$ & Simulation time(sec) & first nodes death time & Half nodes death time & All nodes death time \\
\hline \multirow{3}{*}{ ISC-EAR } & Mine topology & 633 & 3886 & 6856 \\
\cline { 2 - 5 } & Average topology & 556 & 3145 & 5728 \\
\cline { 2 - 5 } & Random topology & 565 & 3454 & 5935 \\
\hline \multirow{3}{*}{ IGreedy } & Mine topology & 524 & 2856 & 4622 \\
\cline { 2 - 5 } & Average topology & 511 & 2865 & 5453 \\
\cline { 2 - 5 } & Random topology & 505 & 2488 & 3956 \\
\hline
\end{tabular}

See table 2, in the same simulation conditions, the ISC-EAR routing algorithm at the first nodes death time, half node death time and the all nodes death time is much long than Igreedy algorithm. More as you can see, different topologies mine topology than other topology. For example, at the mine topology ISC-EAR algorithm half death nodes time than the Igreedy algorithm increased by $24 \%$, the all nodes dead time increased by $20 \%$. Demonstrate design of the ISC-EAR routing algorithm extended the network survival time, and have good balance and effectiveness.

\subsection{Network energy consumption}

Network energy consumption is defined the total energy consumption in the network. The greater the energy consumption, survival time is shorter of network.

Table 3 shows the random topology, average topology and mine (tunnel) topology at the ISC-EAR routing algorithm and the IGreedy routing algorithm test results for network energy consumption.

Table 3 Network energy consumption

\begin{tabular}{|c|l|c|c|c|}
\hline Routing algorithm & Simulation time(sec) & $\mathbf{1 0 0 0}$ & $\mathbf{2 0 0 0}$ & $\mathbf{3 0 0 0}$ \\
\hline \multirow{3}{*}{ ISC-EAR } & Mine topology & 28 & 115 & 227 \\
\cline { 2 - 5 } & Average topology & 30 & 131 & 251 \\
\cline { 2 - 5 } & Random topology & 30 & 170 & 291 \\
\hline \multirow{3}{*}{ IGreedy } & Mine topology & 35 & 138 & 286 \\
\cline { 2 - 5 } & Average topology & 38 & 160 & 315 \\
\cline { 2 - 5 } & Random topology & 33 & 205 & 395 \\
\hline
\end{tabular}

From table 3, with the increase of the simulation time, three kinds of network topology of the network energy consumption rising trends. Different topology at the same time simulation contrast, mine topology network energy consumption at least. The same topology at the same time simulation contrast, ISC-EAR routing algorithm is less than the benchmark algorithm. For example, in the simulation time for 3000 seconds of mine topology energy consumption to $26 \%$ lower than the IGreedy routing algorithm. 


\subsection{Average energy of node}

Average energy of node is defined as the network energy consumption within a certain time and the ratio of the amount of data transferred, reflects the network node load balance, load is balanced, the longer the network survival time.

Table 4 shows the random topology, average topology and mine (tunnel) topology at the ISC-EAR routing algorithm and the IGreedy routing algorithm test results for average energy of node.

\begin{tabular}{|c|l|c|c|c|}
\hline \multicolumn{9}{|c|}{ Table 4 Average energy of node } \\
\hline \multirow{3}{*}{ Routing algorithm } & Simulation time(sec) & $\mathbf{1 0 0 0}$ & $\mathbf{2 0 0 0}$ & $\mathbf{3 0 0 0}$ \\
\hline \multirow{3}{*}{ ISC-EAR } & Mine topology & 2.81 & 3.11 & 3.49 \\
\cline { 2 - 5 } & Average topology & 2.92 & 3.21 & 3.59 \\
\cline { 2 - 5 } & Random topology & 2.95 & 3.35 & 3.79 \\
\hline \multirow{3}{*}{ IGreedy } & Mine topology & 3.01 & 3.39 & 4.89 \\
\cline { 2 - 5 } & Average topology & 3.06 & 3.42 & 4.36 \\
\cline { 2 - 5 } & Random topology & 3.18 & 3.6 & \\
\hline
\end{tabular}

From table 4, with the increase of the simulation time, three kinds of network topology at the node average energy consumption rising trends; Different topology at the same time simulation contrast, mine topology node average energy consumption minimum. For example, in the simulation time for 3000 seconds of mine topology the ISC-EAR algorithm is $25 \%$ lower than the IGreedy routing algorithm at random topology.

\section{Conclusion}

In this paper ISC-EAR routing algorithm proposed, for analysis using the $3 \mathrm{~d}$ wireless sensor network (WSN), at variety of topology, various technical indicators are compared with the benchmark algorithm Igreedy has obvious advantages. Has good advancement and research value, can be in various kinds of 3d wireless sensor network application.

\section{References}

[1] Rahman A. U., Hasbullah H., Sama N.U. Efficient Energy Utilization Through Optimum Number of Sensor Node Distribution in Engineered Corona-Based (ONSD-EC) Wireless Sensor Network [J]. Wireless Personal Communications , 2013, 73(3) 1227-1243.

[2] Garcia M., Sendra S., Lloret J. and Canovas A. Saving energy and improving communications using cooperative group-based Wireless Sensor Networks [J]. Telecommunication Systems, 2013, 52(4) 2489-2502.

[3] Abdallah A.E., Fevens T., Opatrny J, Stojmenovic I. Power-aware semi-beaconless 3D georouting algorithms using adjustable transmission range for wireless ad hoc and sensor networks [J]. Ad Hoc Networks, 2010, 8(1) 15-29.

[4] Mehdi A., Mohammad A., Masoud S. A Rate-Distortion Based Aggregation Method Using Spatial Correlation for Wireless Sensor Networks[J]. Wireless Personal Communications, 2013, 71(3)1837-1877.

[5] H.F. Jiang, J.S. Qian, W. Peng. Energy efficient sensor placement for tunnel wireless sensor network in underground mine[A].Proceedings of the $2^{\text {nd }}$ International Conference on Power Electronics and Intelligent Transportation System[C], 2009. 